\title{
ARrion $\mid$ Uso de preservativo e vulnerabilidades para infecções sexualmente transmissíveis em comunidades quilombolas: estudo descritivo, Sergipe, 2016-2017* \\ doi: 10.1590/S1679-49742021000200011
}

\author{
Condom use and vulnerabilities to sexually transmitted infections in quilombola \\ communities: a descriptive study, Sergipe, Brazil, 2016-2017 \\ Uso de preservativo y vulnerabilidades a las infecciones de transmisión sexual en \\ comunidades quilombolas: un estudio descriptivo, Sergipe, Brasil, 2016-2017
}

\author{
Taciana Silveira Passos ${ }^{1}$ - (1) orcid.org/0000-0002-5312-095X \\ Marcos Antonio Almeida-Santos ${ }^{1}$ - (1) orcid.org/0000-0003-0622-6257 \\ Aline Barreto Hora' ${ }^{1}$ - (D) orcid.org/0000-0002-3930-6475 \\ Cristiane Costa da Cunha Oliveira' ${ }^{1}$ - (1) orcid.org/0000-0003-1439-7961 \\ 'Universidade Tiradentes, Pós-Graduação em Saúde e Ambiente, Aracaju, SE, Brasil
}

\section{Resumo}

Objetivo: Analisar a frequência do uso de preservativos segundo fatores de vulnerabilidade para infecções sexualmente transmissíveis em comunidades quilombolas de Sergipe, Brasil. Métodos: Estudo transversal descritivo, realizado entre 2016 e 2017. Utilizou-se questionário estruturado, com questões sociodemográficas e comportamentais; foram realizados testes rápidos de HIV e sífilis. Comparações entre variáveis categóricas foram realizadas pelo teste exato de Fisher. Resultados: Entre os 367 indivíduos de 14 comunidades, a maioria apresentava baixa escolaridade (72,8\%), encontrava-se sem trabalho (59,7\%) e possuía parceiro sexual fixo $(90,7 \%)$. Falta de acesso a insumos e informações de prevenção compuseram a vulnerabilidade programática dos indivíduos. Houve maior proporção de uso inconsistente do preservativo com parceiro fixo $(90,1 \%)$, em indivíduos que relataram falta de acesso à informação $(\mathrm{p}=0,001)$ e uso inconsistente com parceiro eventual $(\mathrm{p}<0,001)$. Conclusão: A frequência de uso do preservativo com parceiro fixo foi significativamente proporcional ao uso com parceiro eventual e ao acesso a informação preventiva.

Palavras-chave: HIV; Sífilis; Preservativos; Vulnerabilidade em Saúde; Grupo com Ancestrais do Continente Africano; Epidemiologia Descritiva.

*Artigo derivado de dissertação de mestrado intitulada 'Vulnerabilidades para HIV/AIDS e Sífilis em População Quilombola do Nordeste Brasileiro', defendida por Taciana Silveira Passos junto ao Programa de Pós-Graduação em Saúde e Ambiente, Universidade Tiradentes, em 2017. O estudo recebeu apoio financeiro da Coordenação de Aperfeiçoamento de Pessoal de Nível Superior (Capes) (Código do Financiamento 001).

Endereço para correspondência:

Cristiane Costa da Cunha Oliveira - Avenida Murilo Dantas, n 300, Farolândia, Aracaju, SE, Brasil. CEP: 49032-490

E-mail: criscunhaoliva@yahoo.com.br 


\section{Introdução}

A cada dia, em todo o mundo, surge mais de 1 milhão de novos casos de infecções sexualmente transmissíveis (ISTs) curáveis entre pessoas de 15 a 49 anos, conforme dados disponibilizados pela Organização Mundial da Saúde em 2019. ${ }^{1} 0$ uso correto e consistente de preservativos é um método eficaz para reduzir a transmissão de ISTs, conquanto a adoção efetiva desse método dependa da disposição, motivação e habilidade própria, e do parceiro ou parceira. ${ }^{2} \mathrm{Em}$ determinados ambientes tradicionais, a exemplo das comunidades quilombolas, o tema pode ser motivo de constrangimento e, muitas vezes, é visto como um tabu. ${ }^{3}$

Observa-se baixa adesão ao uso consistente do preservativo no ambiente das comunidades quilombolas, em diferentes contextos regionais, incluindo os estados do Pará, ${ }^{3}$ Maranhão, ${ }^{4}$ Goiás e Mato Grosso do Sul. ${ }^{5}$ Entre os motivos para o não uso desse instrumento de prevenção, destaca-se a falta de percepção do risco de contrair ISTs.

\section{Observa-se baixa adesão ao uso consistente do preservativo no ambiente das comunidades quilombolas, em diferentes contextos regionais}

Além do conjunto de vulnerabilidades individuais relacionadas aos comportamentos sexuais de risco, os fatores sociais atuam como fortes preditores. ${ }^{6}$ No que se refere às desigualdades étnicas e raciais no Brasil, a situação dos quilombolas merece atenção especial, haja vista o histórico de vulnerabilidade dessas comunidades. ${ }^{?}$

Os resultados dos estudos de comportamento sexual e vulnerabilidade para ISTs em afrodescendentes brasileiros devem servir de subsídio ao desenho e implementação de políticas públicas, tanto gerais quanto específicas. Contudo, eles são escassos no que concerne às ISTs em comunidades quilombolas no estado de Sergipe. ${ }^{3-5,8}$ Nesse sentido, o presente estudo pretende contribuir para a ampliação do conhecimento sobre a situação de vulnerabilidade (individual, social e programática) dessa população, comumente sub-representada em estudos epidemiológicos na área temática da saúde sexual.
Sob essa perspectiva, este trabalho teve como objetivo analisar a frequência do uso de preservativos segundo fatores de vulnerabilidade para infecções sexualmente transmissíveis em comunidades quilombolas de Sergipe, Brasil.

\section{Métodos}

Trata-se de um estudo do tipo transversal descritivo, realizado em 14 comunidades quilombolas do estado de Sergipe, região Nordeste do Brasil, entre 2016 e 2017.

Segundo a Fundação Cultural Palmares, no Brasil, até setembro de 2017, foram emitidos 2.526 certificados para 3.010 comunidades quilombolas, 35 delas assentadas em Sergipe, distribuídas entre 11 municípios. ${ }^{9}$

As comunidades quilombolas selecionadas localizam-se nas bacias hidrográficas dos rios Japaratuba, São Francisco, Costeiro do Sapucaia e Sergipe, e nos territórios do Médio Sertão Sergipano, Grande Aracaju, Baixo São Francisco Sergipano e Leste Sergipano.

Foram elegíveis para o estudo adultos com idade de 18 anos ou mais, residentes em comunidades quilombolas do estado. Indivíduos com diagnóstico clínico de comprometimento mental grave, registrado em prontuário da unidade básica de saúde (UBS) vinculada à comunidade, e aqueles que nunca tiveram relações sexuais, foram excluídos.

A amostra do estudo foi estratificada entre as comunidades quilombolas, identificadas a partir dos dados registrados pelo Instituto Nacional de Colonização e Reforma Agrária de Sergipe: famílias dos territórios adscritos, cadastradas como remanescentes de comunidades quilombolas. Uma vez criado um banco de dados dos territórios e dos indivíduos cadastrados, utilizou-se o procedimento de amostragem aleatória sem substituição.

0 desfecho do estudo foi o uso de preservativo (sempre; frequentemente; às vezes; nunca) com as alternativas dicotomizadas (uso inconsistente; uso consistente), sendo considerado 'uso consistente' apenas quando indivíduos elegeram a alternativa 'sempre'. Aos entrevistados também foi questionado o uso do preservativo conforme 0 tipo de parceria em que estavam envolvidos: parceiros fixos ou parceiros eventuais.

As demais variáveis estudadas incluíram as dimensões da vulnerabilidade propostas por Jonathan Mann et al. e por Ayres (este, no Brasil): $:^{10-11}$ 
a) Vulnerabilidade social

- Sexo (masculino; feminino);

- Raça/cor da pele autodeclarada (branco; pardo; preto);

- Nível de escolaridade (sem estudo ou com ensino fundamental incompleto; ensino fundamental completo/ensino médio incompleto; ensino médio completo/ensino superior incompleto; ensino superior completo);

- Classificação econômica, categorizada segundo o Critério Brasileiro de Classificação Econômica da Associação Brasileira de Empresas de Pesquisa (ABEP) de 2016 (categorias: A; B1; B2; C1; C2; $\mathrm{D} / \mathrm{E}) ;^{12}$

- Renda familiar (dicotomizada pela mediana: até $\mathrm{R} \$ 600$ reais; mais de $\mathrm{R} \$ 600) ; \mathrm{e}$

- Situação de trabalho atual (sem trabalho; com trabalho).

b) Vulnerabilidade individual

- Idade (anos: 18 a 39; 40 a 59; 60 a 101), cujos pontos de corte foram definidos com base na mediana da distribuição da amostra;

- Uso de drogas (usuário ocasional ou de baixo risco; risco ou uso nocivo; alta chance de dependência), baseado na versão brasileira do instrumento Alcobol, Smoking and Substance Involvement Screening Test (ASSIST); ${ }^{13}$

- Parceiro usuário de drogas (sim; não);

- Histórico de ISTs do entrevistado e do parceiro (presença; ausência);

- Tipos de relações sexuais (oral [sim; não]; vaginal [sim; não]; anal [sim; não]);

- Número de parceiros sexuais nos últimos 12 meses (até cinco; mais de cinco); e

- Idade da primeira relação sexual (menos de 15 anos; 15 ou mais anos).

c) Vulnerabilidade programática

- Acesso a alguma informação sobre prevenção de ISTs nos últimos 12 meses (sim; não);

- Recebimento de insumos - preservativos e gel lubrificante - nos últimos 12 meses (sim; não);

- Local onde realiza controle de saúde (UBS; consultório particular; não realiza);

- Tratamento após apresentar ISTs (sim; não);

- Serviço de saúde onde realizou o tratamento

(UBS; farmácia; não tratou);
- Presença de programas sociais governamentais de prevenção de IST que incluam pessoas do local (sim; não);

- Racismo institucional (discriminação: ausente ou baixa; média; alta), avaliado por uma escala de tipo Likert com cinco pontos, que variam de 1 (nunca) a 5 (sempre), com base no ponto de corte da escala de percepção de discriminação racial em saúde, validada e adaptada à realidade brasileira. $^{14}$

0s dados foram coletados no período de setembro de 2016 a junho de 2017. A equipe de pesquisadores apresentou o projeto a cada líder quilombola e programou as datas mais adequadas para a realização de um evento e coleta de dados, respeitando a ordem cronológica da solicitação de cada líder e a confirmação da disponibilidade de algum espaço público da comunidade: UBS, escolas, centros comunitários etc.

As entrevistas utilizaram-se de um questionário estruturado por itens relacionados às vulnerabilidades social, individual e programática; elas foram realizadas individualmente, em locais públicos previamente agendados ou na residência do entrevistado quando era impossível a este deslocar-se até o local estabelecido para a pesquisa.

Após a entrevista, os participantes foram convidados a fazer o teste rápido para o HIV e a sífilis. Aqueles que assentiram foram encaminhados para aconselhamento individual (aconselhamento pré-teste) e, posteriormente, em uma sala reservada, submeteram-se a coleta de material sorológico. 0s resultados dos testes foram informados em outro aconselhamento individual (aconselhamento pós-teste), complementado pela educação em saúde e recomendações de prevenção pautadas em cada diagnóstico.

A infecção pelo HIV foi identificada pelos testes rápidos BioManguinhos HIV-1\&2 e Abon. Aplicou-se 0 teste Alere na detecção de anticorpos para Treponema pallidum, o que permitiu identificar a ocorrência de sífilis independentemente de ser precoce, diagnosticada até dois anos de vida, ou tardia, quando já se passaram mais de dois anos do contato efetivo com a bactéria. A realização dos testes seguiu as orientações das portarias do Ministério da Saúde n 34, de 28 de julho de 2005, e n ${ }^{0} 3.242$, de 30 de dezembro de 2011.

Todos os participantes com resultado positivo foram encaminhados à UBS responsável pela cobertura de 
seu território, onde apresentaram os formulários apropriados: Ficha de Encaminhamento para HIV e Sífilis; uma via da ficha clínica; e a ficha Fique Sabendo, do Departamento de IST, Aids e Hepatites Virais de Sergipe.

0 cálculo do tamanho amostral pautou-se em um poder de $80 \%$, alpha bicaudal de 0,05 e mínima diferença detectável entre grupos de $15 \%$, totalizando um mínimo de 333 indivíduos a serem entrevistados. Foram acrescidos $10 \%$ a este valor, para compensar eventuais dados faltantes e perdas.

Antes de sua aplicação pelos pesquisadores, o questionário passou por um processo de validação de conteúdo do qual se encarregaram cinco peritos reconhecidos, dedicados às interdisciplinariedades de Saúde e Ambiente, Saúde Coletiva e Saúde Sexual, além de servir ao treinamento dos entrevistadores, 20 estudantes de graduação e pós-graduação na área da Saúde. Tais estratégias buscaram diminuir o viés de aferição.

A análise estatística foi realizada com uso do software Stata versão 15.0. As variáveis contínuas foram descritas em médias e desvios-padrão (DP); e as variáveis categóricas, em valor absoluto e percentual, acompanhadas de intervalo de confiança a 95\% $\left(\mathrm{IC}_{95 \%}{ }^{\circ}\right.$, estimado pelo método "exato" binomial. Para comparações da distribuição do uso de preservativo entre as demais variáveis que compuseram a vulnerabilidade para IST, utilizou-se o teste exato de Fisher. 0 p-valor bicaudal $<0,050$ foi considerado critério de significância estatística. Utilizou-se a relação sexual com parceiro fixo como referência, por representar a maior parcela da população.

0 projeto da pesquisa foi aprovado pelo Comitê de Ética em Pesquisa da Universidade Tiradentes (CEP/ UNIT) em seu Parecer $n^{0}$ 1.685.357, de 18 de agosto de 2016, e também pela Secretaria de Estado da Saúde de Sergipe e Secretarias Municipais de Saúde cujas comunidades estariam envolvidas no estudo. Todos os participantes assinaram o Termo de Consentimento Livre e Esclarecido.

\section{Resultados}

A amostra foi composta por 367 indivíduos de 14 comunidades quilombolas de Sergipe, com idades entre 18 e 101 anos (média de 44,81-DP=17,46). Entre os 333 indivíduos $(90,7 \%)$ que mantinham relações sexuais com parceiro fixo, $9,9 \%$ utilizavam preservativo de forma consistente nessa forma exclusiva de relação.
No que diz respeito aos 191 (52,1\%) que mantinham relações com parceiro eventual, $15,7 \%$ utilizavam preservativo de forma consistente. Dentro desse mesmo quadro, 42,7\% mantinham relações sexuais tanto com parceiro fixo quanto com parceiros eventuais, sendo que entre esses indivíduos com as duas formas de parceria, $90,6 \%$ realizavam sexo desprotegido com ambos os tipos de parceiro - fixo e eventual.

0 perfil dos participantes, de acordo com a vulnerabilidade social, é descrito na Tabela 1 . Houve predominância de negros - pardos e pretos $(93,2 \%)$-, classe econômica D/E (75,4\%), sexo feminino (73,0\%), indivíduos sem estudo ou com ensino fundamental incompleto $(72,8 \%)$, com renda familiar até $\mathrm{R} \$ 600$ $(66,5 \%)$ - equivalente a aproximados US\$190 por na ocasião da entrevista - e sem trabalho (59,7\%).

0 consumo de álcool foi relatado por $61,1 \%$ da população e, segundo a classificação do ASSIST, 17,2\% dos entrevistados apresentavam risco ou uso nocivo ou alta chance de dependência (Tabela 1). 0 uso de outras drogas psicoativas na vida - e.g., maconha, cocaína, hipnóticos e inalantes - foi mencionado por cerca de $10 \%$ da população.

A prevalência de sífilis encontrada foi de 3,3\%, não houve resultado positivo nos testes para HIV e $6,8 \%$ relataram antecedentes de ISTs. Quanto aos fatores de vulnerabilidade individual por comportamento sexual, $28,9 \%$ iniciaram a atividade sexual com menos de 15 anos de idade e 3,3\% referiram ter mais de cinco parceiros (Tabela 2).

Com relação à vulnerabilidade programática para IST, os seguintes participantes haviam-se submetido a testes de detecção de HIV e sífilis alguma vez na vida: $41,1 \%$ haviam realizado o teste para o HIV, e 35,4\%, o teste para sífilis. A falta de acesso a algum tipo de informação de prevenção para IST foi relatado por $41,1 \%$ dos entrevistados, e 82,8\% não receberam insumos nos últimos 12 meses. No que diz respeito aos programas sociais governamentais de prevenção de ISTs que incluíssem pessoas do local, 79,6\% referiram não contar com esse recurso na comunidade e $83,9 \%$ utilizavam a UBS para controle de saúde. A maioria - 95,4\% - referiu baixa ou nenhuma percepção de haver sofrido atos de racismo nos serviços de saúde (Tabela 3).

Observaram-se maiores proporções de uso inconsistente do preservativo com parceiro fixo em indivíduos que relataram falta de acesso à informação $(\mathrm{p}=0,001)$, como também entre indivíduos com parceiro eventual $(\mathrm{p}<0,001)$ (Tabela 4). Verificou-se, ademais, uso 
Tabela 1 - Frequências absoluta e relativa das variáveis que compõem os fatores a vulnerabilidade social para infecções sexualmente transmissiveis em comunidades quilombolas de Sergipe, 2016-2017

\begin{tabular}{lcc}
\hline Variável & $\mathbf{n}$ & $\%$ \\
\hline Sexo & 268 & 73,0 \\
\hline Masculino & 99 & 27,0 \\
Feminino & 342 & 93,2 \\
\hline Raça/cor da pele autodeclarada & 25 & 6,8 \\
\hline Negra (pardos e pretos) & & \\
Branca & 214 & 72,8 \\
\hline Escolaridade & 53 & 27,2 \\
\hline Sem estudo ou com ensino fundamental incompleto & & \\
Ensino fundamental completo, ou ensino médio/ensino superior completos ou incompletos & 244 & 66,5 \\
\hline Renda familiar & 123 & 33,5 \\
\hline Até R\$ 600 & & \\
Mais de R\$̧ 600 & 90 & 24,6 \\
\hline Classificação econômica ${ }^{2}$ & 277 & 75,4 \\
\hline Classe B1 a C2 & & \\
Classe D/E & 219 & 59,7 \\
\hline Situação de trabalho atual & 148 & 40,3 \\
\hline Sem trabalho & & \\
Com trabalho & 143 & 38,9 \\
\hline Consumo de álcool & 161 & 43,9 \\
\hline Não consome & 63 & 17,2 \\
\hline Uso ocasional ou de baixo risco & & \\
Uso de risco ou uso nocivo/alta chance de dependência & & \\
\hline
\end{tabular}

a) Critério Brasileiro de Classificação Econômica da Associação Brasileira de Empresas de Pesquisa (ABEP) de 2016 (categorias: A; B1; B2; C1; C2; D/E).

consistente do preservativo com parceiro fixo em indivíduos do sexo feminino $\left(78,8 \%\right.$ - $\left.\mathrm{IC}_{95 \%} 61,3 ; 89,6\right)$ e sem trabalho $\left(69,7 \%\right.$ - $\left.\mathrm{IC}_{95 \%}, 51,9 ; 83,0\right)$, embora as diferenças não fossem significativas.

\section{Discussão}

0 uso inconsistente do preservativo foi referido por aproximadamente um em cada dez moradores das comunidades quilombolas de Sergipe. Pouco mais da metade dos respondentes também possuía parceiro eventual e praticava sexo sem preservativo em ambas as situações. Houve maior proporção de uso consistente do preservativo com parceiro fixo entre indivíduos que também o utilizavam de forma consistente com parceiro eventual, e igualmente, como esperado, entre aqueles que tiveram acesso à informação preventiva. Baixo nível de escolaridade e más condições socioeconômicas foram os principais fatores de sua vulnerabilidade social e, cumpre lembrar e destacar, trata-se da mesma realidade presente em muitas comunidades do país, 0 que enseja uma reflexão mais profunda sobre questões de acesso à saúde com equidade.

Os resultados apresentados devem ser interpretados considerando-se algumas limitações. Uma delas é a carência de estudos nacionais sobre o tema, principalmente das populações quilombolas. A mesma escassez desses estudos também implicou a necessidade de, na discussão dos resultados da pesquisa, incluir estudos de diferentes abordagens metodológicas. 0 delineamento transversal descritivo, por sua vez, impossibilita 0 estabelecimento de uma temporalidade para as análises. Vale ressaltar que os indivíduos foram questionados sobre práticas anteriores ao momento da entrevista, sendo possível que suas respostas sofressem 
Tabela 2 - Frequências absoluta e relativa das variáveis que compõem os fatores de vulnerabilidade individual para infecções sexualmente transmissíveis em comunidades quilombolas de Sergipe, 2016-2017

\begin{tabular}{|c|c|c|}
\hline Fatores de vulnerabilidade individual & $\mathbf{n}$ & $\%$ \\
\hline \multicolumn{3}{|l|}{ Faixa etária (anos) } \\
\hline $18-39$ & 148 & 40,3 \\
\hline $40-59$ & 139 & 37,9 \\
\hline $60-101$ & 80 & 21,8 \\
\hline \multicolumn{3}{|l|}{ Histórico de IST (entrevistado) } \\
\hline Sim & 25 & 6,8 \\
\hline Não & 342 & 93,2 \\
\hline \multicolumn{3}{|l|}{ Histórico de IST (parceiro) } \\
\hline Sim & 4 & 1,1 \\
\hline Não & 363 & 98,9 \\
\hline \multicolumn{3}{|l|}{ Parceiro usuário de drogas } \\
\hline Sim & 20 & 5,4 \\
\hline Não & 347 & 94,6 \\
\hline \multicolumn{3}{|l|}{ Idade da primeira relação sexual } \\
\hline 15 ou mais anos de idade & 261 & 71,1 \\
\hline Menos de 15 anos de idade & 106 & 28,9 \\
\hline \multicolumn{3}{|c|}{ Número de parceiros sexuais nos últimos 12 meses } \\
\hline Até 5 & 64 & 96,7 \\
\hline Mais de 5 & 256 & 3,3 \\
\hline \multicolumn{3}{|l|}{ Tipo de relação sexual } \\
\hline \multicolumn{3}{|l|}{ Vaginal } \\
\hline Sim & 346 & 94,3 \\
\hline Não & 21 & 5,7 \\
\hline \multicolumn{3}{|l|}{ Anal } \\
\hline Sim & 28 & 7,6 \\
\hline Não & 339 & 92,4 \\
\hline \multicolumn{3}{|l|}{ Oral } \\
\hline Sim & 12 & 3,3 \\
\hline Não & 355 & 96,7 \\
\hline
\end{tabular}

a influência do viés de memória. Adicionalmente, existe a possibilidade de algumas respostas corresponderem ao padrão "socialmente aceitável".

Era de se esperar a proporção significativa do uso consistente do preservativo com parceiro eventual e com parceiro fixo. Teoricamente, usuários consistentes de preservativo com parceiro fixo apresentariam menos risco de contrair IST, ${ }^{6}$ porque não deixariam de se prevenir da mesma forma com o parceiro eventual.
Quanto ao uso inconsistente com parceiro fixo e parceiro eventual, outros estudos, publicados entre 2009 e 2017 , tendo como objeto comunidades quilombolas do Norte, Nordeste e Centro-0este do país, já haviam mencionado essa característica, inclusive nas situações de múltiplos parceiros. ${ }^{3-5}$

A informação insuficiente pode ser um fator agravante do risco de infecção, ao promover desconhecimento sobre 0 método preventivo e, 
Tabela 3 - Frequências absoluta e relativa das variáveis que compõem os fatores de vulnerabilidade programática para infecções sexualmente transmissíveis em comunidades quilombolas de Sergipe, 2016-2017

\begin{tabular}{|c|c|c|}
\hline Fatores de vulnerabilidade programática & $\mathbf{n}$ & $\%$ \\
\hline \multicolumn{3}{|l|}{ Teste de HIV realizado alguma vez na vida } \\
\hline Sim & 151 & 41,1 \\
\hline Não & 216 & 58,9 \\
\hline \multicolumn{3}{|l|}{ Teste de sífilis realizado alguma vez na vida } \\
\hline Sim & 130 & 35,4 \\
\hline Não & 237 & 64,6 \\
\hline \multicolumn{3}{|c|}{ Acesso a alguma informação sobre prevenção de IST nos últimos 12 meses } \\
\hline Sim & 216 & 58,9 \\
\hline Não & 151 & 41,1 \\
\hline \multicolumn{3}{|c|}{ Recebimento de insumos - preservativos e gel lubrificante - nos últimos 12 meses } \\
\hline Não & 304 & 82,8 \\
\hline Sim & 63 & 17,2 \\
\hline \multicolumn{3}{|l|}{ Local onde realiza controle de saúde } \\
\hline Unidade básica de saúde & 308 & 83,9 \\
\hline Consultório particular & 39 & 10,6 \\
\hline Não realiza & 20 & 5,5 \\
\hline \multicolumn{3}{|l|}{ Tratamento após apresentar IST } \\
\hline Serviço de saúde & 16 & 66,7 \\
\hline Farmácia & 3 & 12,5 \\
\hline Não tratou & 5 & 20,8 \\
\hline \multicolumn{3}{|c|}{ Programas sociais e governamentais de prevenção de IST que incluam pessoas do local } \\
\hline Não & 292 & 79,6 \\
\hline Sim & 75 & 20,4 \\
\hline \multicolumn{3}{|c|}{ Percepção de discriminação racial nos serviços de saúde } \\
\hline Ausente ou baixa & 110 & 95,4 \\
\hline Média & 16 & 4,3 \\
\hline Alta & 1 & 0,3 \\
\hline
\end{tabular}

consequentemente, disseminação das mais variadas crendices relacionadas ao preservativo. ${ }^{15-16}$ Essa situação é ainda mais grave no contexto de comunidades quilombolas, muitas vezes afastadas das unidades de saúde, em sua maioria de nível socioeconômico desfavorável, menos dotadas de recursos para sua educação, saúde e transporte.

0 perfil sociodemográfico dos participantes foi similar ao encontrado em outras pesquisas realizadas em comunidades quilombolas: população predominantemente negra, sem estudo ou com ensino fundamental incompleto, e sem trabalho. Observa-se a predominância de famílias em condições econômicas menos favoráveis, situação reportada em relatos de pesquisa publicados entre os anos de 2009 e 2020, sobre populações quilombolas dos estados da Bahia, ${ }^{17-19}$ Pará, ${ }^{3}$ Maranhão, ${ }^{4}$ Goiás e Mato Grosso do Sul. ${ }^{5}$

Estudos norte-americanos, realizados na década de $1990^{20}$ e no decorrer dos últimos dez anos, ${ }^{6}$ concluíram que pessoas com um nível socioeconômico alto tendem a apresentar um risco muito menor de adquirir uma IST. 0 nível educacional tem papel fundamental 
Tabela 4 - Análise da distribuição do uso de preservativo com parceiro fixo segundo os fatores de vulnerabilidade para infecções sexualmente transmissíveis em comunidades quilombolas de Sergipe, 2016-2017

\begin{tabular}{|c|c|c|c|c|c|c|}
\hline \multirow{3}{*}{ Fatores de vulnerabilidade } & \multicolumn{4}{|c|}{ Resultado para o uso } & \multirow{3}{*}{$\begin{array}{l}\text { Total } \\
\text { n (\%) }\end{array}$} & \multirow{3}{*}{ p-valor } \\
\hline & \multicolumn{2}{|c|}{ Uso inconsistente } & \multicolumn{2}{|c|}{ Uso consistente } & & \\
\hline & n (\%) & $\mathrm{IC}_{95 \%}{ }^{\mathrm{a}}$ & n (\%) & IC $_{95 \%}{ }^{\mathrm{a}}$ & & \\
\hline Sexo & & & & & & 0,077 \\
\hline Feminino & $215(71,7)$ & 66,$2 ; 76,5$ & $26(78,8)$ & 61,$3 ; 89,6$ & $241(72,4)$ & \\
\hline Masculino & $85(28,3)$ & 23,$4 ; 33,7$ & $7(21,2)$ & 10,$3 ; 38,6$ & $92(27,6)$ & \\
\hline Sabe ler e escrever & & & & & & 0,383 \\
\hline Sim & $175(58,3)$ & 52,$6 ; 63,8$ & $24(72,7)$ & 54,$9 ; 85,3$ & $199(59,8)$ & \\
\hline Não & $125(41,7)$ & 36,$1 ; 47,3$ & $9(27,3)$ & 14,$6 ; 45,0$ & $134(40,2)$ & \\
\hline Sem trabalho & & & & & & 0,059 \\
\hline Sim & $175(58,3)$ & 52,$6 ; 63,8$ & $23(69,7)$ & 51,$9 ; 83,0$ & $198(59,5)$ & \\
\hline Não & $125(41,7)$ & 36,$1 ; 47,3$ & $10(30,3)$ & 16,$9 ; 48,0$ & $135(40,5)$ & \\
\hline Consumo de álcool & & & & & & 0,791 \\
\hline Não consome & $115(38,3)$ & 32,$9 ; 43,9$ & $9(27,3)$ & 14,$6 ; 45,0$ & $124(37,2)$ & \\
\hline Usuário ocasional ou de baixo risco & $131(43,7)$ & 38,$1 ; 49,3$ & $19(57,6)$ & 40,$1 ; 73,2$ & $150(45,1)$ & \\
\hline Usuário de risco/uso nocivo & $45(15,0)$ & 11,$3 ; 19,5$ & $5(15,1)$ & 6,$3 ; 3,2$ & $50(15,0)$ & \\
\hline Usuário de alto risco & $9(3,0)$ & 1,$5 ; 5,6$ & - & - & $9(2,7)$ & \\
\hline Faixa etária (anos) & & & & & & 0,825 \\
\hline $18-39$ & $118(39,3)$ & 33,$9 ; 45,0$ & $18(54,5)$ & 37,$3 ; 70,6$ & $136(40,8)$ & \\
\hline $40-59$ & $116(38,7)$ & 33,$2 ; 44,3$ & $13(39,4)$ & 24,$1 ; 56,9$ & $129(38,8)$ & \\
\hline $60-101$ & $66(22,0)$ & 17,$6 ; 27,0$ & $2(6,1)$ & 1,$47 ; 21,6$ & $68(20,4)$ & \\
\hline Antecedentes de IST & & & & & & 0,396 \\
\hline Sim & $23(7,7)$ & 5,$1 ; 11,2$ & - & - & $23(6,9)$ & \\
\hline Não & $277(92,3)$ & 88,$7 ; 94,8$ & $33(100,0)$ & - & $310(93,1)$ & \\
\hline Preservativo com parceiro even & & & & & & $<0,001$ \\
\hline Uso inconsistente & $154(90,6)$ & 85,$2 ; 94,2$ & $3(20,0)$ & 6,$3 ; 48,3$ & $157(84,9)$ & \\
\hline Uso consistente & $16(9,4)$ & 5,$8 ; 14,8$ & $12(80,0)$ & 51,$7 ; 93,7$ & $28(15,1)$ & \\
\hline Recebimento de insumos & & & & & & 0,185 \\
\hline Sim & $47(51,4)$ & 11,$9 ; 20,2$ & $10(5,6)$ & 16,$9 ; 48,0$ & $57(17,1)$ & \\
\hline Não & $253(84,3)$ & 79,$7 ; 88,0$ & $23(69,7)$ & 51,$9 ; 83,0$ & $276(82,9)$ & \\
\hline Acesso à informação em saúde s & & & & & & 0,001 \\
\hline Sim & $177(59,0)$ & 49,$2 ; 68,1$ & $29(87,9)$ & 80,$0 ; 92,9$ & $206(61.9)$ & \\
\hline Não & $123(41,0)$ & 31,$8 ; 50,8$ & $4(12,1)$ & 7,$1 ; 19,9$ & $127(38,1)$ & \\
\hline
\end{tabular}

a) $I_{955 ;}$ : intervalo de confiança de $95 \%$; b) Teste exato de Fisher. 
na capacidade adquirida de compreender as informações fornecidas, contempladas nos programas de prevenção, e responder a elas. De acordo com os referidos estudos, populações com, no mínimo, ensino fundamental completo estão menos suscetíveis a contrair IST. Estes achados corroboram a situação de vulnerabilidade às IST identificada por estes autores nas comunidades quilombolas de Sergipe.

A maior proporção de mulheres a utilizar o preservativo com parceiro fixo pode ser reflexo de sua insegurança quanto à fidelidade dele, prevenção de gravidez ou maior preocupação com a saúde. Sabe-se que, para muitos casais, o sexo desprotegido pode ser interpretado como um sinal de intimidade e confiança, resultando em uma barreira psicológica, não só para o uso do preservativo, senão, também, para a comunicação sobre sexo seguro em geral. ${ }^{2}$ Contudo, as autoras de um estudo datado de 2016, realizado com mulheres quilombolas do estado do Amazonas, observaram baixo uso do preservativo entre a população sexualmente ativa, independentemente do seu estado civil. Mesmo assim, a maioria das mulheres entrevistadas reconheceu no preservativo um instrumento seguro de prevenção de IST. $^{3}$

A porcentagem de participantes com relato de consumo alcoólico de alto risco foi superior à encontrada por um estudo realizado em 2011, em comunidades quilombolas da Bahia (11\%), ${ }^{17}$ conquanto se deva levar em consideração que o estudo não utilizou 0 ASSIST como base, e sim uma classificação por número de doses consumidas semanalmente. 0 consumo excessivo de bebidas alcoólicas está associado a um aumento da probabilidade de diagnóstico de alguma IST. Alguns pesquisadores têm associado o consumo do álcool a múltiplas parcerias sexuais e, como consequência, ao uso inconsistente do preservativo, conferindo maior risco de transmissão do HIV. ${ }^{21,22}$

Embora a prevalência de sífilis tenha sido menor do que $10 \%$, ela foi três vezes maior que a encontrada por um estudo de base populacional realizado em parturientes no Brasil ${ }^{23}$ como proxy para a população geral $(0,89 \%)$. Vale ressaltar que a sífilis é considerada um marcador de sexo desprotegido, e pode potencializar a infecção pelo HIV. ${ }^{24,25}$

Os fatores relacionados à dificuldade no acesso a testes para HIV e sífilis, insumos, informações e atividades de prevenção e assistência à saúde fizeram parte da dimensão da vulnerabilidade programática das comunidades quilombolas. A esses achados acrescentam-se os de um estudo de 2010, realizado em comunidades quilombolas de todo o território nacional, no qual se mostraram as mesmas dificuldades de acesso aos serviços de prevenção às IST. ${ }^{8}$ Trata-se de condições importantes para o sucesso das ações de prevenção. Portanto, espera-se que o contato com informações sobre redução do risco de IST, a disponibilidade de insumos e de testes diagnósticos tornará os indivíduos mais preparados para práticas sexuais mais seguras. ${ }^{26,27}$

Paralelamente à realização dos testes para HIV e sífilis, o uso dos serviços públicos de saúde pode diminuir a vulnerabilidade programática. As ações dos programas sociais também são de extrema importância: de acordo com uma pesquisa realizada no ano de 2017, em Coimbra, Portugal, após a intervenção de um programa educacional para redução dos comportamentos sexuais de risco, os indivíduos alcançados tiveram maior probabilidade de usar preservativo na última relação sexual com parceiro estável (razão de chances de 4,61 - $\left.\mathrm{IC}_{95 \%} 1,31 ; 16,20\right)$ e de realizar 0 teste para 0 HIV (razão de chances de 7,59 - $\mathrm{IC}_{95 \%} 3,33 ; 17,35$ ). ${ }^{28}$

A maioria dos entrevistados realizavam o controle de saúde nas UBS. Outro estudo, focado nas comunidades quilombolas do sudoeste da Bahia, em 2013, mostrou dificuldade de acesso e subutilização dos serviços públicos de saúde, aos quais apenas $57,1 \%$ de seus moradores recorriam. ${ }^{18}$ Embora, no plano formal, as comunidades tenham acesso à Estratégia Saúde da Família (ESF), ela não se tem efetivado no cotidiano quilombola, devido às barreiras de acesso. ${ }^{8,19}$ Observou-se, nas comunidades quilombolas de Sergipe, que 0 acesso às UBS é difícil, devido à distância e falta de profissionais. Ademais, para algumas dessas comunidades, o serviço era apenas volante.

A maioria dos indivíduos teve percepção de discriminação racial ausente ou baixa, na Saúde, o que, teoricamente, diminuiria a possibilidade de afastamento do serviço de saúde por sofrer discriminação. No entanto, é importante notar que alguns indivíduos relataram percepção média e alta de racismo institucional na saúde. Destaca-se que a consolidação da ideia do racismo estrutural na formação da desigualdade social brasileira se deu, e se dá, de forma não linear, sob fortes contestações, inflexões e vários 
entraves no caminho ${ }^{29}$ Inclusive, não se descarta a hipótese de compreensão distorcida dos conceitos acerca de racismo, preconceito e discriminação racial entre a população geral. Além disso, a própria dificuldade de acesso pode representar mais uma barreira para 0 indivíduo responder a esse quesito.

0 s resultados apresentados delineiam um perfil de vulnerabilidades individuais, sociais e programáticas para prevenção de IST nas comunidades quilombolas de Sergipe. A frequência de uso do preservativo com parceiro fixo foi diretamente proporcional a seu uso com parceiro eventual e ao acesso à informação para prevenção. 0s resultados reforçam a necessidade de se fortalecerem estratégias que viabilizem a consolidação de práticas de educação sexual, mediante políticas individuais e coletivas mais eficazes,

\section{Referências}

1. Organização Mundial da Saúde (OMS). Organização Pan-Americana de Saúde (OPAS). A cada dia, há 1 milhão de novos casos de infecções sexualmente transmissíveis curáveis [Internet]. Brasília: OPAS; 2019 [citado 2017 ago 31]. Disponível em: https:/www.paho.org/bra/index. php?option $=$ com_content\&view $=$ article\&id $=5958$

2. Ajayi AI, Ismail KO, Akpan W. Factors associated with consistent condom use: a cross-sectional survey of two Nigerian universities. BMC Public Health [Internet]. 2019 Dec [cited 2021 Jan 6];19(1):1207. Available from: https://doi.org/10.1186/s12889-019-7543-1

3. Nascimento VB, Nascimento Martins NV, Ciosak SI, Nichiata LY, Santos Oliveira JS, Bezerra LO, et al. Vulnerabilidades de mulheres quilombolas no interior da Amazônia às infecções sexualmente transmissíveis: um relato de experiência. Interdiscip J Health Educ [Internet]. 2017 Jan-Jul [cited 2021 Jan 6];2(1):68-73. Disponível em: https://doi.org/10.4322/ijhe.2016.029

4. Cardoso RLS. Vulnerabilidades às DSTs/Aids entre jovens de uma comunidade quilombola do município de Turiaçu-MA [dissertação]. São Luís: Universidade Federal do Maranhão; 2011. Disponível em: https:/tedebc.ufma.br/jspui/handle/tede/1080

5. Nascimento LB, Santos Carneiro MA, Teles SA, Lopes CL, Silva Reis NR, Costa Silva ÁM, et al. Prevalência de infecção por HTLV-1 em quilombos remanescentes no Brasil Central. Rev Soc Bras Med Trop [Internet]. 2009 expandindo os serviços prestados pela Saúde às comunidades quilombolas de Sergipe.

\section{Contribuição dos autores}

Passos TS e Hora AB contribuíram com a concepção e delineamento do estudo, interpretação dos resultados e redação do conteúdo do manuscrito. Almeida-Santos JP contribuiu com a concepção e delineamento do estudo, análise estatística dos resultados e revisão crítica do conteúdo do manuscrito. Oliveira CCC contribuiu com a concepção e delineamento do estudo, interpretação dos dados e revisão crítica do conteúdo do manuscrito. Todos os autores aprovaram a versão final do manuscrito e são responsáveis por todos os seus aspectos, incluindo a garantia de sua precisão e integridade. dez [citado 2021 jan 6];42(6):657-60. Disponível em: https://doi.org/10.1590/S0037-86822009000600009

6. Bajaj S, Shravan R, Enebeli S, Nsohtabien H, Andkhoe M, et al. Factores de riesgo en Canadá para enfermedades de transmisión sexual y sus variaciones. Med Soc [Internet]. 2016 [citado 2021 jan 6];11(2):5865. Disponível em: https://www.medicinasocial. info/index.php/medicinasocial/article/view/943

7. Silva ER. Comunidades remanescentes quilombolas no Brasil: construção identidária, desafios e perspectivas na luta pela regularização de seus territórios. Projeto História [Internet]. 2016 [citado 2021 jan 6];56:378-92. Disponível em: https://revistas. pucsp.br/index.php/revph/article/view/26863

8. Silva MJG, Lima FSS, Hamann EM. Uso dos serviços públicos de saúde para DST/HIV/aids por comunidades remanescentes de Quilombos no Brasil. Saúde Soc [Internet]. 2010 [citado 2021 jan 6];19(2):109-20. Disponível em: https://doi. org/10.1590/S0104-12902010000600011

9. Fundação Cultural Palmares (BR). Comunidades quilombolas [internet]. Brasília: Fundação Cultural Palmares; 2017 [citado 2017 jul 21]. Disponível em: http://www.palmares.gov.br/?page_id=88

10. Ayres JRCM, França-Júnior I, Calazans GJ, Saletti-Filho HC. 0 conceito de vulnerabilidade e as práticas de saúde: novas perspectivas e desafios. In: Czeresnia D, Freitas CM. Promoção 
da saúde: conceitos, desafios, tendências. 2. ed. Rio de Janeiro: Fiocruz; 2016. p. 121-44.

11. Mann J. Aids no mundo. Rio de Janeiro: Relume Dumará; 1993.

12. Kamakura W, Mazzon JA. Socioeconomic stratification criteria and classification tools in Brazil. Rev Adm Empresas [Internet]. 2016 [cited 2021 Jan 6];56(1):55-70. Available from: https://doi.org/10.1590/S0034-759020160106

13. Henrique IF, De Micheli D, Lacerda RB, Lacerda LA, Formigoni ML. Validation of the Brazilian version of alcohol, smoking and substance involvement screening test (ASSIST). Rev Assoc Med Bras [Internet]. 2004 [cited 2021 Jan 6];50(2):199-206. Available from: https://doi.org/10.1590/S0104-42302004000200039

14. Miranda RDS. Racismo no Contexto da Saúde: um estudo psicossociológico [tese]. João Pessoa (PB): Universidade Federal da Paraíba; 2015. Disponível em: https://repositorio.ufpb.br/ jspui/handle/tede/7688?locale=pt_BR

15. Nascimento EGC, Fernandes C, Marília AA, João C. Adesão ao uso da camisinha: a realidade comportamental no interior do nordeste do Brasil. Rev Salud Pública [Internet]. 2017 [citado 2021 jan 6];19(1):71-80. Disponível em: https://doi.org/10.15446/rsap.v19n1.44544

16. Uribe-Alvarado JI, Bahamon-Muñeton MJ, ReyesRuíz L, Trejos-Herrera AM, Alarcon-Vásquez Y. Percepción de autoeficacia vs. rechazo del uso del condón en las prácticas sexuales de mujeres y hombres jóvenes. Psicogente [Internet]. 2017 [citado 2021 jan 6];20(37):25-35. Disponible en: https://doi.org/10.17081/psico.20.37.2416

17. Cardoso LGV, Melo AP, Cesar CC. Prevalência do consumo moderado e excessivo de álcool e fatores associados entre residentes de Comunidades Quilombolas de Vitória da Conquista, Bahia, Brasil. Ciên Saúde Coletiva [Internet]. 2015 [citado 2021 jan 6];20(3):809-20. Disponível em: https://doi. org/10.1590/1413-81232015203.12702014

18. Gomes K0, Reis EA, Guimarães MDC, Cherchiglia ML. Utilização de serviços de saúde por população quilombola do Sudoeste da Bahia, Brasil. Cad Saúde Pública [Internet]. 2013 [citado 2021 jan 6];29(9):1829. Disponível em: https:// doi.org/10.1590/0102-311X00151412

19. Pereira RN, Mussi RFF, Rocha RM Acesso e utilização dos serviços de saúde por quilombolas contemporâneos baianos. Rev ABPN [Internet]. 2020 fev [citado 2021 jan 6];12(31). Disponível em: https:// abpnrevista.org.br/index.php/site/article/view/739

20. Wasserheit JN, Aral SO. The dynamic topology of sexually transmitted disease epidemics: implications for prevention strategies. J Infect Dis [Internet]. 1996 [cited 2021 Jan 6];174(Suppl 2):S201-13. Available from: https://doi. org/10.1093/infdis/174.Supplement_2.S201

21. Kermode M, Sono CZ, Songput CH, Devine A. Falling through the cracks: a qualitative study of HIV risks among women who use drugs and alcohol in Northeast India. BMC Int Health Hum Rights [Internet]. 2013 [cited 2021 Jan 6];13(9):1-8. Available from: https://doi.org/10.1186/1472-698X-13-9

22. Choudhry V, Agardh A, Stafstrom M, Ostergren P0. Patterns of alcohol consumption and risky sexual behavior: a cross-sectional study among Ugandan university students. BMC Public Health [Internet]. 2015 [cited 2021 Jan 6];14(128):1-11. Available from: https://doi.org/10.1186/1471-2458-14-128

23. Cunha ARC. Sífilis em parturientes do Brasil: prevalência e fatores associados, 2010-2011 [dissertação]. Brasília (DF): Universidade de Brasília; 2015. Disponível em: http://bdtd.ibict.br/vufind/ Record/UNB_f183ca35107a5525c5bdb1b7b1df939a

24. Shilaih M, Marzel A, Braun DL, Scherrer AU, Kovari $\mathrm{H}$, Young J, et al. Factors associated with syphilis incidence in the HIV-infected in the era of highly active antiretrovirals. Medicine [Internet]. 2017 [cited 2021 Jan 6];96(2):e5849. Available from: https://doi.org/10.1097/MD.0000000000005849

25. Dai W, Luo Z, Xu R, Zhao G, Tu D, Yang L, Wang F, et al. Prevalence of HIV and syphilis co-infection and associated factors among non-commercial men who have sex with men attending a sexually transmitted disease clinic in Shenzhen, China. BMC Infect Dis [Internet]. 2017 [cited 2021 Jan 6];17(86). Available from: https://doi.org/10.1186/s12879-017-2187-1

26. Wasserheit JN, Aral S0. The dynamic topology of sexually transmitted disease epidemics: implications for prevention strategies. J Infect Dis [Internet]. 1996 [cited 2021 Jan 6];174(Suppl 2):S201-13. Available from: https:/doi. org/10.1093/infdis/174.Supplement_2.S201

27. La Croix JM, Pellowski JA, Lennon CA, Johnson BT. Behavioural interventions to reduce sexual risk for HIV in heterosexual couples: a meta-analysis. 
Sex Transm Infect [Internet]. 2013 [cited 2021 Jan 6];89(8):620-7. Available from: https:// doi.org/10.1136/sextrans-2013-051135

28. Oliveira ACGDPC, Caramelo F, Patrício M, Camarneiro AP, Cardoso SM, Pita JR. Impacto de um programa de intervenção educativa nos comportamentos sexuais de jovens universitários. Rev Enf Ref
[Internet]. 2017 [citado 2021 jan 6];4(13):71-82. Available from: https://doi.org/10.12707/RIV17022

29. Oliveiva D0, Brandão WN, Araújo Madeira MZ. Justiça racial e direitos humanos de povos e comunidades tradicionais. Revista Katálysis [Internet]. 2020 maioago [citado 2021 jan 6];23(2):317. Disponível em: https://doi.org/10.1590/1982-02592020v23n2p317

\section{Abstract}

objective: To analyze frequency of condom use according to vulnerability factors for sexually transmitted infections in quilombola communities in Sergipe state, Brazil. Methods: This was a descriptive crosssectional study carried out in 2016-2017. A structured questionnaire with sociodemographic and behavioral questions was used; rapid HIV and syphilis tests were performed. Comparisons between categorical variables were performed using Fisher's exact test. Results: Among the 367 individuals from 14 communities, the majority had low levels of education (72.8\%), were not working (59.7\%) and had a stable sex partner (90.7\%). Lack of access to prevention supplies and information accounted for respondent programmatic vulnerability. There was a higher proportion of inconsistent condom use with a stable partner (90.1\%) among individuals who reported lack of access to information ( $p=0.001)$ and inconsistent use with a casual partner $(p<0.001)$. Conclusion: Frequency of condom use with a stable partner was significantly proportional to condom use with a casual partner and access to information on prevention.

Keywords: HIV; Syphilis; Condoms; Health Vulnerability; African Continental Ancestry Group; Epidemiology, Descriptive.

\section{Resumen}

Objetivo: Analizar la frecuencia de uso de preservativo según factores de vulnerabilidad a infecciones de transmisión sexual en comunidades quilombolas de Sergipe, Brasil. Métodos: Estudio descriptivo transversal realizado entre 2016-2017. Se utilizó un cuestionario estructurado con aspectos sociodemográficos y de comportamiento; y se realizaron pruebas rápidas de VIH y sífilis. Las comparaciones entre variables categóricas se realizaron mediante la prueba exacta de Fisher. Resultados: Entre las 367 personas de 14 comunidades, la mayoría eran de baja escolaridad (72,8\%), sin trabajo (59,7\%) y tenían pareja sexual fija (90,7\%). La falta de acceso a insumos e información de prevención constituyó la vulnerabilidad programática de las personas. Hubo una mayor proporción de uso inconsistente del preservativo con una pareja estable $(90,1 \%)$ en las personas que informaron falta de acceso a la información $(p=0,001)$ y uso inconsistente con una pareja ocasional $(p<0,001)$. Conclusión: La frecuencia del uso de preservativo con pareja estable fue significativamente proporcional al uso con pareja casual y al acceso a información preventiva.

Palabras clave: VIH; Sifilis; Condones; Vulnerabilidad en Salud; Grupo de Ascendencia Continental Africana; Epidemiología Descriptiva.

Recebido em 02/08/2020

Aprovado em 09/10/2020

\footnotetext{
Editora associada: Bárbara Reis-Santos - (1) orcid.org/0000-0001-6952-0352 Editora científica: Taís Freire Galvão - (1) orcid.org/0000-0003-2072-4834 Editora geral: Leila Posenato Garcia - (1) orcid.org/0000-0003-1146-2641
} 